\title{
Experimental and Numerical Based Defect Detection in a Model Combustion Chamber through Machine Learning
}

\author{
Henrik von der Haar, Panagiotis Ignatidis, Friedrich Dinkelacker \\ Institute of Technical Combustion, Leibniz Universität Hannover, \\ An der Universität 1, 30823 Garbsen, Germany
}

\begin{abstract}
A disturbed combustion process in an aircraft engine has an impact on the internal flow and leads to specific irregularities in the species distribution in the exhaust jet. Measuring this distribution provides information about the combustion state and offers the possibility to reduce the engine down-time during inspection. The approach has the potential to improve the resource management as well as the availability and safety of the system. Aim of the research project is to evaluate the state of an aircraft engine by analyzing the emission field in the exhaust jet and using a support vector machine (SVM) algorithm for automatic defect detection and allocation.
\end{abstract}

\section{NOMENCLATURE}

Symbols

$\begin{array}{lll}\mathrm{b} & = & \text { bias } \\ \mathrm{D} & = & \text { number of features } \\ \mathrm{M} & = & \text { feature } \\ \mathrm{P} & = & \text { power } \\ \mathrm{W} & = & \text { normal vector of hyperplane } \\ \mathrm{X}_{\mathrm{i}} & = & \text { sample } \\ \lambda & = & \text { air-fuel equivalence ratio }\end{array}$

\begin{tabular}{ccl}
\multicolumn{2}{l|}{ Abbreviations } & \\
\hline Ref & $=$ & reference class \\
$\mathrm{Sh}=$ & shifted burner \\
$\mathrm{Ti}=$ & tilted burner \\
$\mathrm{CFD}=$ & Computational Fluid Dynamics \\
$\mathrm{CRC}=$ & Collaborative Research Center \\
$\mathrm{FTIR}=$ & Fourier-Transform Infrared Spectrometer \\
$\mathrm{MRO}=$ & Maintenance, Repair and Overhaul \\
$\mathrm{RANS}=$ & Reynolds-Averaged Navier-Stokes \\
$\mathrm{RBF}=$ & Radial Basis Function Kernel \\
$\mathrm{SAS}=$ & Scale-Adaptive Simulation \\
$\mathrm{SVM}=$ & Support Vector Machine
\end{tabular}

\section{INTRODUCTION AND SPECIFIC OBJECTIVES}

The current work demonstrates the combination of this approach on a simplified model ring combustion chamber with eight burners, where possible engine failures can be implemented. The combination of experimental determination of the spatial exhaust gas pattern, numerical simulations and machine learning algorithms are used to recognize the pattern in the exhaust jet automatically.

Manuscript Received on December 17, 2020

Review Completed on November 16, 2021
The present work consists of two steps: In the first step, a generic study on the model combustion chamber is done. The setup features the flexible variation of the operating point or the alignment of one of eight premixed swirl burners, to create an irregularity, which corresponds to a defect in a jet engine. The influence on the distribution and concentration of the species field for different exemplary defects is investigated experimentally and numerically. In the second step, the measurement data are used to implement and test a support vector machine for pattern recognition at this given setup. This works well for either a general failure detection with a simple one-class SVM approach and also for selective multi-class SVM methods to recognize the different failure types. Additionally it is investigated, if the training data of the exhaust gas measurement could be replaced by the data sets from the numerical simulations with CFD. This may reduce cost and time to implement such a system and allows to train defects, which cannot be measured before. The results demonstrate the potential of the approach and support the feasibility of an automatic defect detection in a combustion chamber of a real aircraft engine. Paul et al. [1] show a possibility for applying a Support Vector Machine for novelty detection in jet engines using vibration data. The method in this paper uses the distribution of exhaust gas components to detect defects in the combustion chamber as well to classify and locally assign them.

Maintenance of aircraft engine systems is so far based on regular MRO schedules, which are oriented on the shortest possible time between potential failures. In this research project, a methodology is sought to enable a diagnosis during operation, to determine informed decisions on the timing and extent of regeneration measures at an early stage. This may allow to extend or shorten operation cycles on demand before expensive repair is needed. Furthermore, detailed pre-knowledge on potential failure sources inside the engine would allow to plan and prepare the necessary regeneration process in advance before the disassembly of the engine, which would lead to higher efficiency.

In previous numerical studies [2] and [3] it has been proved that defects in combustion chambers influence flow and combustion and can be detected by an exhaust gas analysis. Fig. 1 shows the impact of a fuel nozzle defect on the temperate field and the propagation through the turbine section.

The basic hypothesis of this study is, that errors in the combustion chamber cause significant and unique pattern in the exhaust jet. With the help of the Support Vector Machine method, the hot gas pattern in the exhaust plane is analyzed in such a way that the connection to the defect in the burning chamber is found automatically, which causes this pattern. For the investigation of this connection between burning chamber defect and exhaust gas pattern, we follow a combined experimental and numerical approach (Fig. 1). 


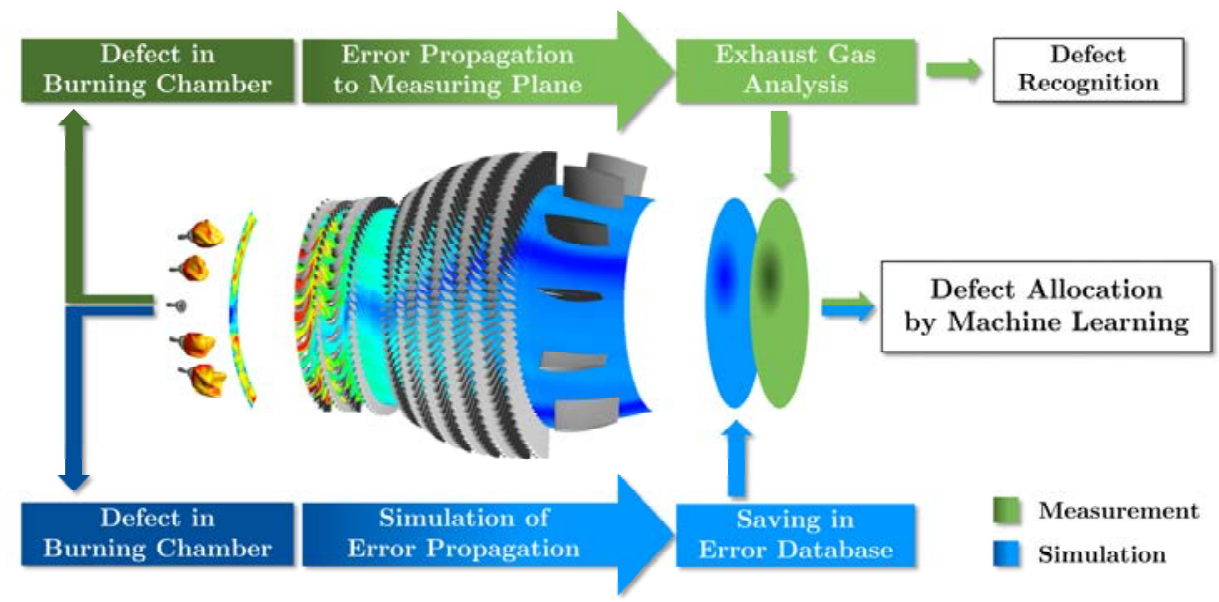

Fig. 1: Methodical concept of defect detection by machine learning

The influences of frequently occurring and economically relevant defects are simulated and stored in an error database (blue path). This process is the defect allocation. If the influence of the defect on the exhaust gas is significant and can be detected by an appropriate measurement system (green path), the measurement results can be assigned to a defect from the database by using classification methods. For a successful defect detection, the used simulation models must be of sufficient quality and the defect influences must be distinguishable.

The defect recognition does not use any databases from previous calculations. With this method, the results from the measurements are only compared with the recorded reference case without any defects. Machine learning can also be used here to find significant deviations in large amounts of data. This procedure can only determine, if there is a defect, but the type of damage is still unknown. Exceptions can be defects that have already occurred several times and have been recorded by measurement. In this case, these data records can be added to a separate database.

In this study, the classification is based solely on the exhaust gas composition, being measured with the FTIR method. The distribution of $\mathrm{CO}_{2}$ correlates strongly with the temperature, so the results can be interpreted also in terms of the measurement of temperature plus species information.

\section{EXPERIMENTAL SETUP}

Since measurements on real aircraft engines are very complex, the experimental and numerical studies have been performed on an atmospheric model combustion chamber, which represents a simplified model of an aircraft engine combustion chamber (Fig. 2).

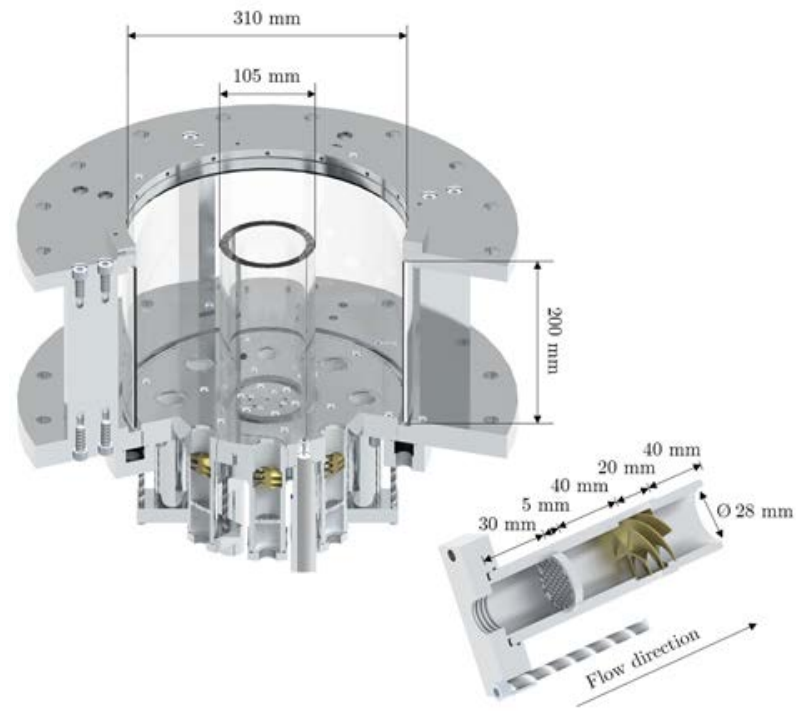

Fig. 2: Model combustion chamber
This combustion chamber allows a detailed investigation of the allocability of combustor defects analyzing patterns in the exhaust jet, as it has easy access for experimental measurement data and can be operated with several different failure cases.

The combustor was designed to investigate the influence of possible defects on the species and temperature distribution in the exhaust plane of the combustor. In the orientation as the combustion chamber can be seen in Fig. 2, the fluid flows from the bottom to the top. The exhaust plane is located right above the glass tube.

The combustor provides the opportunity to simulate various defects, which may occur in an annular combustion chamber of an aircraft engine. The combustion chamber consists of an array of eight premixed-operated swirl-burners, which are arranged in a circle with a diameter of $210 \mathrm{~mm}$. Every burner is composed out of a tube with an inner diameter of $28 \mathrm{~mm}$ in which a swirl generator and a turbulence grid are mounted. The combustion chamber is outwardly and inwardly enclosed by cylindrical quartz glass, which allows optical access. The outer diameter is $310 \mathrm{~mm}$ and the inner diameter is $105 \mathrm{~mm}$. The inner glass ring is cooled by an air flow from the inside. [4]

The gas flow rates of the burners and the cooling flow are controlled by flow meters. To generate a defect in the combustion chamber, one burner can be regulated independently of the other seven. This allows to vary the power and the fuel-air-ratio. Defects can also be generated by changing the positioning of the burner tubes: One burner can be moved clockwise by $20^{\circ}$ on the pitch circle. Another burner can be tilted by $10^{\circ}$ in relation to the flow direction (see Fig. 3). For more information, see also [4] and [5].

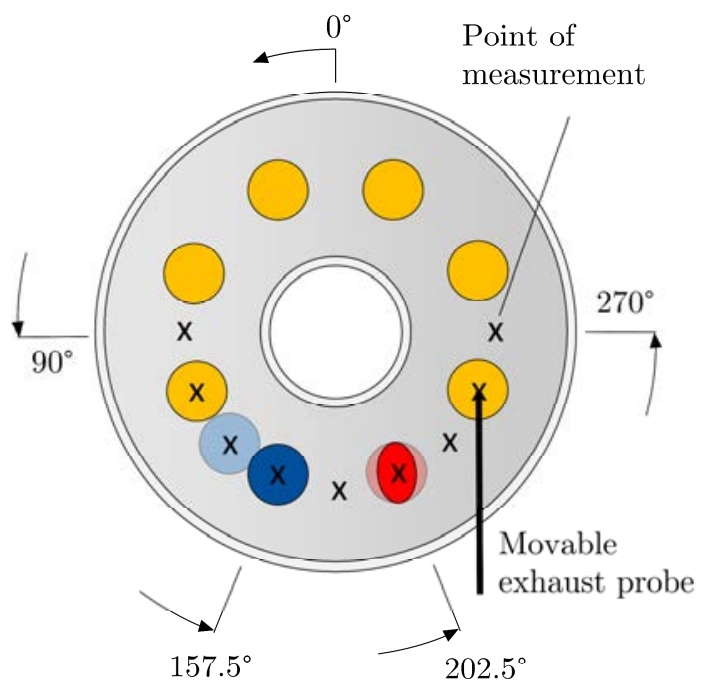

Fig. 3: Schematic top view of the model combustion chamber: Point of measurement (x); manipulated and tiltable burner (red); shiftable burner (blue) 
Table 1: Investigated operating points and defects

\begin{tabular}{|c|c|c|c|c|c|c|}
\hline Name & PSingle & $\lambda_{\text {Single }}$ & $\mathbf{P}_{\text {Array }}$ & $\lambda_{\text {Array }}$ & Shifted (Sh) & Tilted (Ti) \\
\hline Ref-P15ג1.0 (Sh/Ti) & $15 \mathrm{~kW}$ & 1.0 & $7 \times 15 \mathrm{~kW}$ & 1.0 & $\checkmark$ & $\checkmark$ \\
\hline$P 12 \lambda 1.0$ & $12 \mathrm{~kW}$ & 1.0 & $7 \times 15 \mathrm{~kW}$ & 1.0 & - & - \\
\hline P10ג1.0 (Sh/Ti) & $10 \mathrm{~kW}$ & 1.0 & $7 \times 15 \mathrm{~kW}$ & 1.0 & $\checkmark$ & $\checkmark$ \\
\hline P5ג1.0 (Sh/Ti) & $10 \mathrm{~kW}$ & 1.0 & $7 \times 15 \mathrm{~kW}$ & 1.0 & $\checkmark$ & $\checkmark$ \\
\hline$P 0(S h)$ & $0 \mathrm{~kW}$ & - & $7 \times 15 \mathrm{~kW}$ & 1.0 & $\checkmark$ & - \\
\hline P0-Air (Sh/Ti) & $0 \mathrm{~kW}$ & $\infty$ & $7 \times 15 \mathrm{~kW}$ & 1.0 & $\checkmark$ & $\checkmark$ \\
\hline$P 15 \lambda 0.8(\mathrm{Sh} / \mathrm{Ti})$ & $15 \mathrm{~kW}$ & 0.8 & $7 \times 15 \mathrm{~kW}$ & 1.0 & $\checkmark$ & $\checkmark$ \\
\hline$P 15 \lambda 0.9$ & $15 \mathrm{~kW}$ & 0.9 & $7 \times 15 \mathrm{~kW}$ & 1.0 & - & - \\
\hline$P 15 \lambda 1.1$ & $15 \mathrm{~kW}$ & 1.1 & $7 \times 15 \mathrm{~kW}$ & 1.0 & - & - \\
\hline P15ג1.2 (Sh/Ti) & $15 \mathrm{~kW}$ & 1.2 & $7 \times 15 \mathrm{~kW}$ & 1.0 & $\checkmark$ & $\checkmark$ \\
\hline
\end{tabular}

The exhaust gas composition and distribution is determined at the outlet plane using the Fourier-transform infrared spectrometer versa06 (IAG GmbH). FTIR spectrometers are the most widely used spectrometers in the field of infrared spectroscopy. Compared to dispersive devices, they offer the advantages of a better signalto-noise ratio, a short measurement time due to the simultaneous acquisition of the entire spectrum, and a high level of wavenumber accuracy.

The exhaust gas is extracted with a traversable ceramic suction probe with heated pipe. The inner diameter of the pipe is $7.5 \mathrm{~mm}$. The outer diameter is $12.5 \mathrm{~mm}$. The measurement points are positioned on a semicircle and are shown in Fig. 3. The measuring rate is $1 \mathrm{~Hz}$, while the measurement accuracy is specified with $\pm 2 \%$ by the manufacturer. The exhaust gas composition is recorded at each of the nine measurement positions for at least 90 seconds, whereby the first 25 seconds due to the intake and settling process are not taken into account in the evaluation. Concentrations of around 30 different species can be determined with the used spectrometer. The current work focuses on the gas components $\mathrm{CO}_{2}$, $\mathrm{CO}$ and NO. Previous investigations [6] have shown that these species are appropriate and offer the greatest potential to detect and distinguish defects in combustion chambers, while further components do not add additional value here.

It should be noted that the model combustion chamber is simplified with respect to a real aircraft engine, as no turbine behind the burning chamber is included. On a first glance, this may be seen a strong simplification, but CFD simulations of the mixing of combustor defects generated flow profile within the turbine indicate that failure pattern remain to be visible here. The CFD simulation in Fig. 1 shows an example of the propagation of a cold streak in an aircraft engine, which is caused by a defect burner.

The current study focuses on some typical generic errors in aircraft engines, the deviation of the air and fuel supply of one burner, the loss of thermal power by a blocked fuel line, or a shifted or tilted fuel nozzle (see Table 1). Combinations of different defects

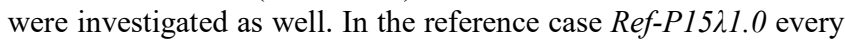
burner has a power of $15 \mathrm{~kW}$ and is operating under stoichiometric conditions. In the first investigation (red marked) the power of one of the eight burner is reduced or totally shut-down, which represents a blockage of the fuel line. In the defect case $P 0$ also the air flow is shut-down, while the air supply is working in the case P0-Air. In a second investigation (blue marked) the air-fuel equivalence ratio $\lambda$ of one burner is varied slightly. Some of these measurements were also repeated $(\checkmark)$ with a shifted or tilted burner. In these cases, a ' $S h$ ' or a ' $T i$ ' is added to the name. In total, one reference case and 22 different defect cases were investigated.

\section{NUMERICAL SETUP}

In addition to the experimental investigations, the processes in the model combustion chamber are evaluated numerically with three-dimensional reacting flow simulations with the CFD method (being based on the ANSYS Fluent 17.2 [7] software). The CFD simulations are especially used for the process to classify defects on the basis of numerical data. The simulation of the flow and combustion processes is divided in two steps. In a first step, the steady flow within the eight combustion tubes (Fig. 4, right) is determined. The tetrahedron mesh (orange) has a comparatively fine resolution and 3.8 million cells due to the complex geometry of the swirl generator. A stationary RANS approach with the SST$\mathrm{k}-\omega$ turbulence model is used to simulate the flow within the eight combustion tubes. The calculations are carried out without combustion.

The flow and turbulence parameters are extracted at the end of the burner tubes and are used as boundary conditions for the transient SAS simulation of the combined ring burning chamber. The separation of the simulation regions enables a significant reduction in computing time, since the flow in the burner tubes is calculated only once. The ring burning chamber is discretized with a hexahedron mesh (blue) with 4.79 million cells and a correspondingly high quality (Fig. 4, left). For the combustion processes the species transport approach with the 2-step mechanism CH4-CM2 was used. The NOx model, which is available as standard in ANSYS Fluent 17.2 is used to determine nitrogen oxides, see [7]. All simulations are performed with a pressure-based solver. A complete mixture of air and methane before entering the combustion tubes is assumed. The time step size of the SAS simulation is $50 \mu \mathrm{s}$. The temperature of the mixture is $20^{\circ} \mathrm{C}$.

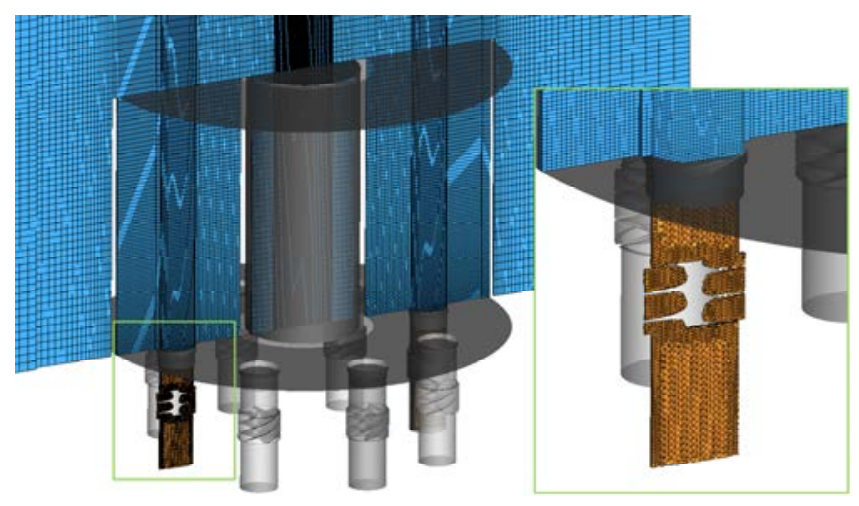

Fig. 4: Used hexahedral (blue) and tetrahedron (orange) meshes of both simulations combined

\section{EXEMPLARY EMISSION PATTERN}

The following diagrams in Fig. 5 show the averaged profile courses at the exit of the model combustion chamber for various defects. The measurement results (left) are based on the FTIR investigations at nine measurement positions in one half of the combustion chamber. The simulated courses (right) were extracted from the simulated species distributions at the same positions. The dashed red line shows the position of the manipulated burner. The error bars and the error bands indicate the $95 \%$ confidence interval. The description of further defects can be found in [8]. 

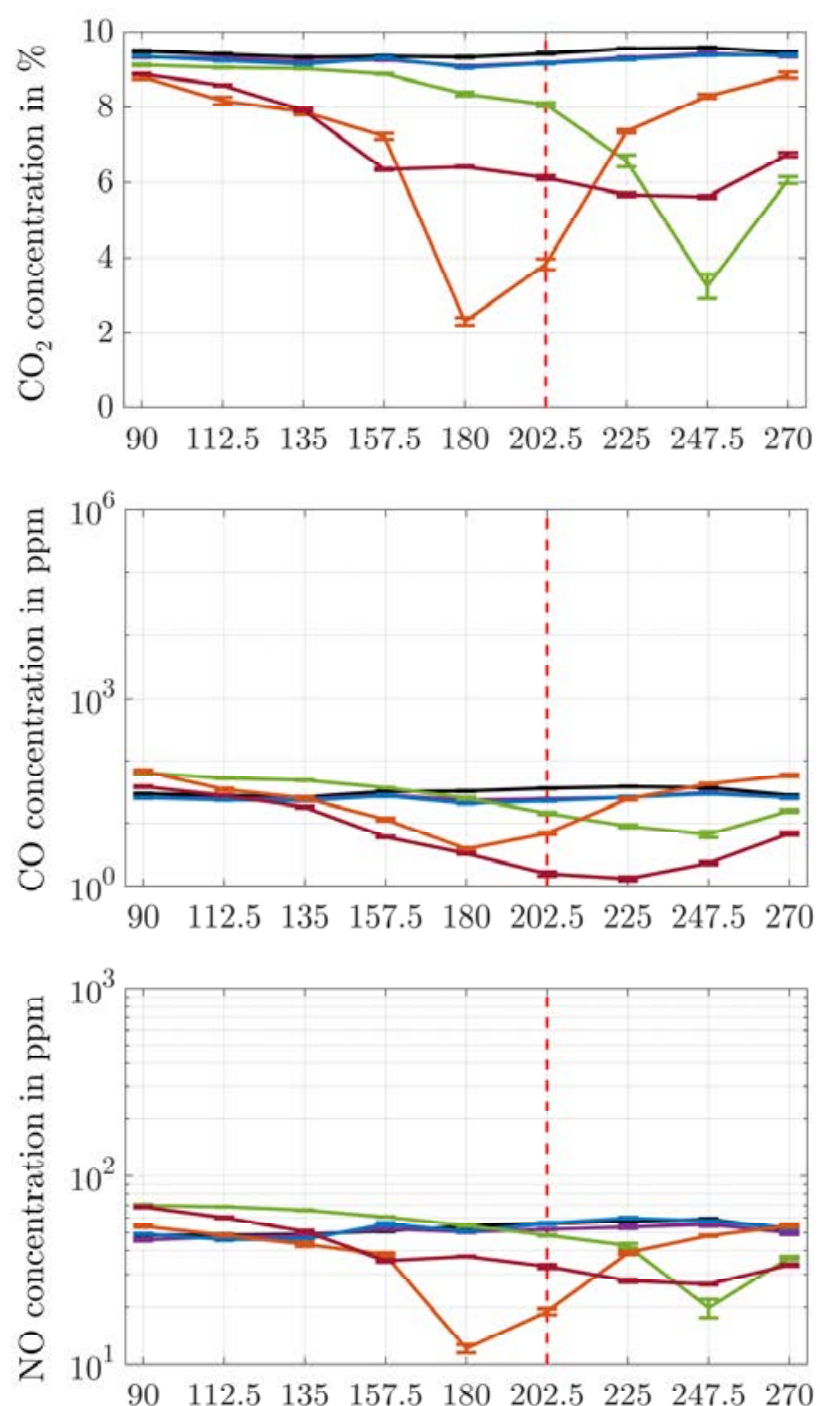

Circumferential position in ${ }^{\circ}$

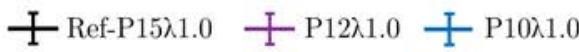
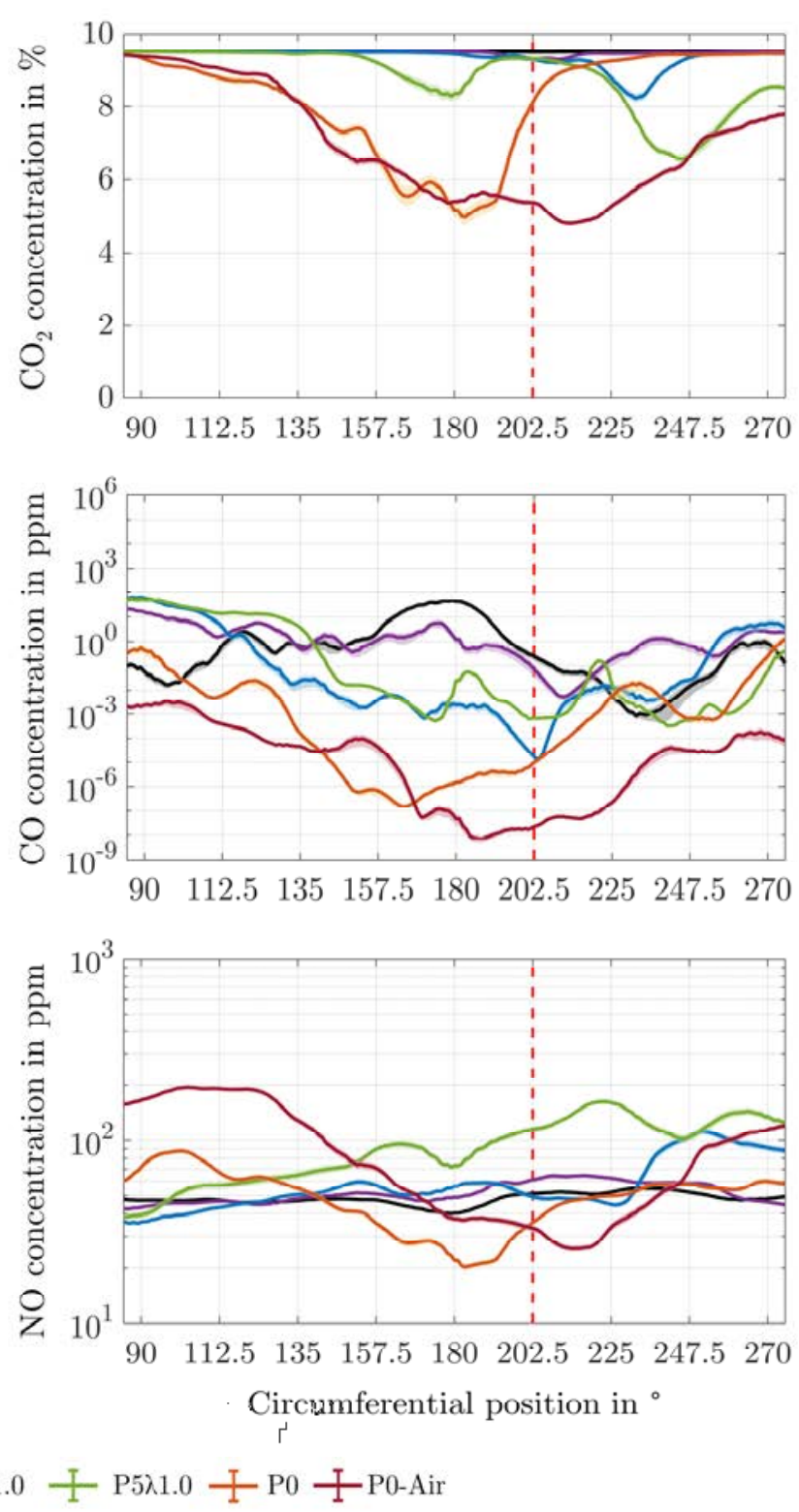

Fig. 5: Circumferential profile of measured (left) and calculated species concentrations (right) at the combustor exit plane

The $\mathrm{CO}_{2}$ concentration for a methane-air combustion under stoichiometric conditions is about $9.51 \%$. For the reference case Ref-P15ג1.0 this value is confirmed very well by both the FTIR measurement and the CFD simulation. The $\mathrm{CO}_{2}$ distributions are constant over the entire range. The profiles of the defect cases also approach this value in the areas, which are not affected by the defect The profiles of both diagrams show great similarity to one another, even if the exact concentrations are not always achieved by the simulation. The defect case $P 0$ with a deactivated burner reduces the $\mathrm{CO}_{2}$ concentration on the left side of the manipulated burner (see dashed line at $202.5^{\circ}$ ). The simulation correctly determines the greatest influence at $180^{\circ}$. The width of the influence is also similar. The presumable reason for the different positions of the concentration minimum are the reduced swirl and flow velocities and the influence of the neighboring burners. The minimum $\mathrm{CO}_{2}$ concentration is overestimated with $5 \%$ compared to $2.3 \%$ in the measurement. The defect case P0-Air with a deactivated fuelsupply shows a broad influence on the $\mathrm{CO}_{2}$ distribution in the exhaust gas. In this case, both the profile and the absolute values of the measurements are met with great agreement by the simulation.

The simulations show fluctuations of the $\mathrm{CO}$ distributions. The simulated and measured influence of defects on the CO distribution profile are similar. The minimum and width of the area of influence are usually correctly determined. The absolute $\mathrm{CO}$ concentrations shows some differences. In the case of P0-Air a minimum concentration of $1 \mathrm{ppm} \mathrm{CO}$ is determined in the measurement and is at the lower limit of the measuring range. The simulation calculates a minimum of $10^{-8} \mathrm{ppm}$. In both cases there is no significant $\mathrm{CO}$ concentration in the exhaust gas, but the value range specified by the simulation cannot be reached by any exhaust gas measurement technology. This fact is problematic for later defect classification. The level of the NO concentration is correctly specified for the reference case at around $40 \mathrm{ppm}$ and corresponds to the measurements. The areas of influence and concentration minima are largely correct. The blocked fuel line (P0-Air) and the inflow of ambient air result in a local excess of oxygen, which oxidizes to NO and leads to a higher NO concentration at about $112.5^{\circ}$ and $270^{\circ}$. The simulation overestimates this process resulting in higher NO concentrations compared with the measurements in this position.

The used simulation models represents the flow and combustion processes in the model combustion chamber to a large extent correctly and are confirmed by the measurement results. The positions of defect influence largely agree with those of the measurements. Overall, the results of the simulation are valid and, in addition to the measurement data, serve as a further data basis for testing the algorithms for pattern recognition. 


\section{SUPPORT VECTOR MACHINE}

The aim of the following study is to check the feasibility to detect a defect and to classify the type of defect from the measured emission pattern. For that the Support Vector Machine (SVM) approach is used. The Support Vector Machine approach has become popular for solving classification tasks. This classification method was introduced in 1992 by Boser et al. [9] and became stateof-the-art due to their fast and simple online application to large scale data sets [10].

The process consists of two steps: the training and the classification of new data. Each data point is described by a set of characteristic features (D of them), such that the data point is presented within a D-dimensional data space. In the first step, a data set of two known classes will be separated by a calculated hyperplane (see Fig. 6 for the simple case of two dimensions). The hyperplane is positioned in the middle between the two canonical hyperplanes (dashed lines). The so-called support vectors will determine the position of these lines, whereby the algorithm is searching for a maximum margin to separate the classes as effectively as possible. In the second step the algorithm is using the hyperplane in order to estimate the class of new and previously unseen data sets. The position of a new data point in relation to the hyperplane determine the class affiliation. [8]

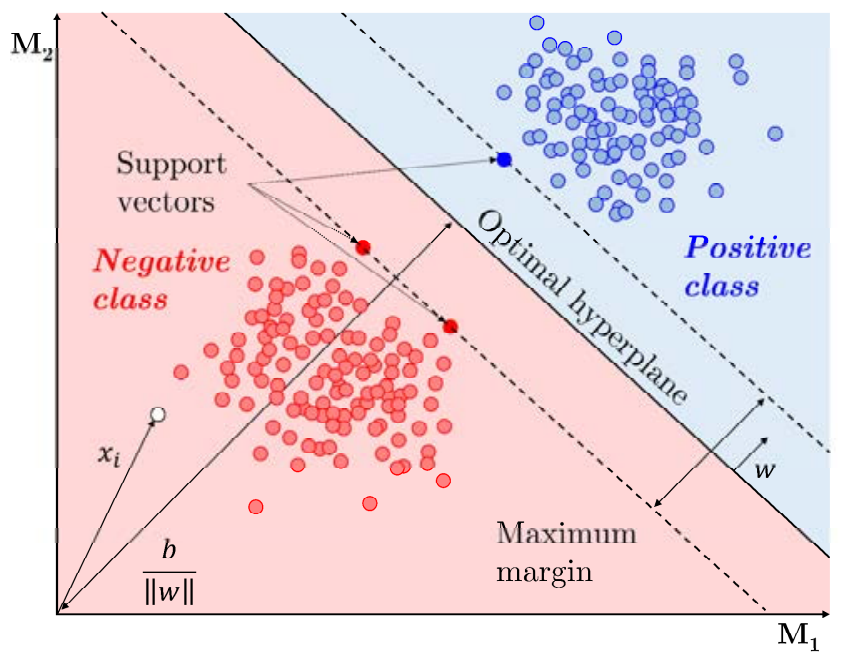

Fig. 6: Example of two linearly separable classes

For the determination of the hyperplane, we assume $\mathrm{N}$ training points are given, which are linearly separable. Each point $x_{i}$ has $\mathrm{D}$ parameters $\left(\mathrm{P}_{1}, \mathrm{P}_{2}, \ldots, \mathrm{P}_{\mathrm{D}}\right)$ and belongs to one of two classes $y_{i}=-1$ or +1 . The hyperplane can be obtained by

$$
w \cdot x+b=0
$$

where vector $w$ is normal to the hyperplane and $b /\|w\|$ is the perpendicular distance from the hyperplane to the origin. After scaling $w$ and $b$ the training data can be described by

$$
\begin{array}{lll}
x_{i} \cdot w+b \geq+1 & \text { for } & y_{i}=+1 \\
x_{i} \cdot w+b \leq-1 & \text { for } & y_{i}=-1
\end{array}
$$

Equation (2) and (3) are combined to

$$
y_{i}\left(w \cdot x_{i}+b\right)-1 \geq 0
$$

The margin between the classes can be described as $1 /\|w\|$. For the determination of the optimal hyperplane the margin between the classes has to be maximized, which is equivalent to minimizing Equation (5) subject to the constraint in (4).

$$
\min \frac{1}{2}\|w\|^{2}
$$

This constrained optimization problem can be written as one equation using the method of Lagrange multipliers [10]:

$$
\mathrm{L}=\frac{1}{2}\|w\|^{2}-\sum_{i=1}^{N} \alpha_{i}\left[y_{i}\left(x_{i} \cdot w+b\right)-1\right]
$$

Substituting the partial derivatives of the Lagrange function

$$
\begin{gathered}
\frac{\partial L_{P}}{\partial w}=0 \rightarrow w=\sum_{i=1}^{N} \alpha_{i} y_{i} x_{i} \\
\frac{\partial L_{P}}{\partial b}=0 \rightarrow 0=\sum_{i=1}^{N} \alpha_{i} y_{i}
\end{gathered}
$$

into Equation (6) results in the dual formulation of the optimization problem

$$
\begin{gathered}
\mathrm{L}_{D}=\sum_{i=1}^{N} \alpha_{i}-\frac{1}{2} \sum_{i, j}^{N} \alpha_{i} \alpha_{j} y_{i} y_{j} x_{i} \cdot x_{j} \\
\alpha_{i} \geq 0 ; \quad \sum_{i, j}^{N} \alpha_{i} y_{i}=0
\end{gathered}
$$

Solving the optimization problem by maximizing $\mathrm{L}_{D}$ leads to the variables $\alpha_{i}$, which are required to determine $w$ and the optimal hyperplane.

Data are often not linearly separable or greater margin can be achieved by allowing to misclassify some points. To allow errors the Equations (4) and (5) may be extended by the slack variable $\xi_{i}$. Additionally the radial basis function kernel (RBF) is used to transform the data set in a higher dimension, where a linear separation is possible.

The SVM algorithm will be used several times to distinguish between more than two classes in multiclass applications. A typical method for multiclass SVM is one-vs-one, which is used here. These topics are not described in the part above, but are used for an efficient algorithm implementation. For further explanations see [11], [12].

\section{IMPLEMENTATION}

Each measured or simulated $\mathrm{CO}_{2}, \mathrm{CO}$ or $\mathrm{NO}$ concentration at every point of investigation is a possible feature for the automatic defect detection. The data are normalized by the algorithms and used without any other modifications. The measurements contain 60 to 120 data points per defect and measurement position. The species distributions were exported from the CFD simulations at every tenth time step, so that the algorithms have at least 450 data points per class and position. The data points are divided into a training $(80 \%)$ and a test data set $(20 \%)$. The MATLAB R2018b [13] functions fitcsvm (one-class learning) and fitcecoc (multi-class learning) with automatic hyperparameter Bayesian optimization are used. The selection of suitable features is based on the eleven investigation cases in Table 2. Each case describes a combination of exhaust gas components that were recorded at different measurement positions and are taken into account in an own SVM.

The specified positions are on the left $\left(180^{\circ}\right)$, in the middle $\left(202.5^{\circ}\right)$ or on the right $\left(225^{\circ}\right)$ above the manipulated burner (see Fig. 3) and were selected on the basis of previous studies. For example, the case $\# 1$ only considers $\mathrm{CO}_{2}$ concentrations at three different positions. The SVM of case \#4 considers the $\mathrm{CO}_{2}, \mathrm{CO}$ and $\mathrm{NO}$ concentration at the position $180^{\circ}$ as features. 
Table 2: Investigation cases with different combinations of features

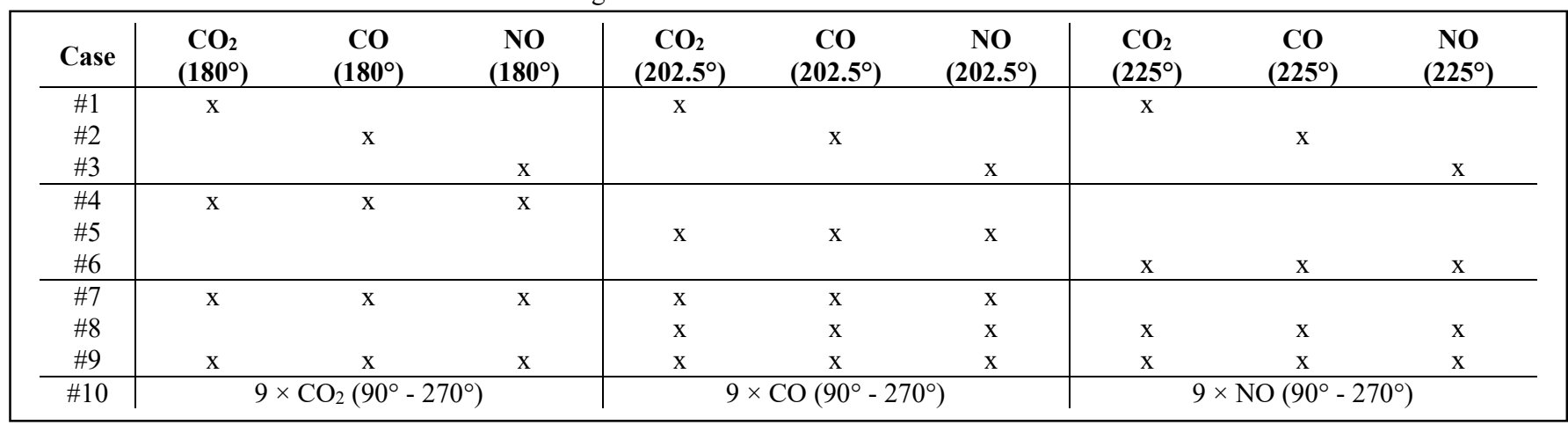

In this paper the features are determined only in a few selected positions. For an evaluation of the entire combustion chamber, the classification must be carried out several times at different positions. The advantage of this method is that several defects can be detected at the same time, which occur at different points in the combustion chamber. The procedure is not carried out in this work, but would be possible straight forward.

\section{RESULTS: ONE-CLASS-SVM}

The classification of only one class is used to detect anomalies in data sets. Here, only the reference case is made available to the SVM algorithm and trained. Test data that lie outside the determined hyperplane belong to the defect class. The main advantage of this approach is that defects can be detected, which have not been trained before. There is no need to create a damage library, but it is not possible to differentiate between defects in this way. The feasibility of this approach is examined in this section.

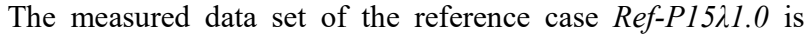
divided into a training $(80 \%)$ and a test data set $(20 \%)$. The data of all defect cases are summarized in one defect class. These data are only used in the SVM test phase to check the classification accuracy. The data set is not available for the algorithm in the learning phase.

Figure 7 shows an extract of the measurement data distribution for the two features $\mathrm{CO}_{2}\left(180^{\circ}\right)$ and $\mathrm{CO}_{2}\left(202.5^{\circ}\right)$. The training data set of the reference class and the test data set of the defect class are shown. Red circled training data points mark the support vectors, which define the hyperplane around the reference class. Furthermore, the determined score is shown as a contour in the diagram. The score defines the class of the data. Test data with a score $>0$ are assigned to the reference class. Data with a score $<0$ are classified as defect. It can be clearly seen that the highest score values are close to the reference class and decrease with increasing distance. The support vectors are distributed evenly around the

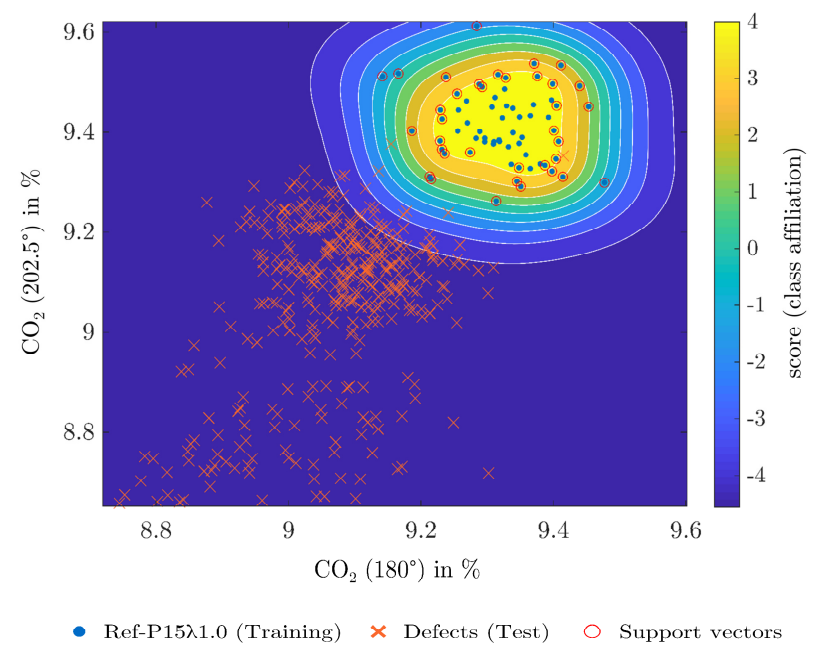

Fig. 7: Extract of the FTIR data distribution of the reference and all defect cases for exemplary features. The score of the one-class SVM determines the class affiliation. training data. The algorithm of the one-class SVM offers the possibility of defining an outlier rate. As a result, the largest outliers of the reference class are neglected, whereby the decision plane move closer to the training data set. This procedure offers the advantage that measurement data from defect engines that are close to the reference class are more likely to be assigned to the defect class. This increases the true-positive-rate (defect is recognized as a defect) at the expense of the true-negative-rate (reference is recognized as a reference) and is particularly preferred for safetycritical systems. The selected outlier-rate is $5 \%$.

The diagram illustrates the good separability of the reference and defect classes. Almost all test data can be correctly assigned with just two features. It should be emphasized once again that only the $\mathrm{CO}_{2}$ concentration of the measurements are plotted, which are only slightly influenced by a defect. The $\mathrm{CO}_{2}$ concentration of the other defects are outside of the diagram area and can be assigned to the defect class with a correspondingly high degree of certainty.

In Figure 8, the proportions of the correctly classified test data are plotted for different features. Each case describes a combination of different exhaust gas concentrations that were recorded at different positions at the combustion chamber outlet. The corresponding characteristics of the respective cases can be found in Table 2. The investigation was carried out for the numerically (a) and experimentally (b) determined data sets. A distinction is made between the success rates of the reference and defect class.

The vast majority of the test cases show good classification results of over $85 \%$ for the test data of the reference case in both studies. The best combinations of features classify about $95 \%$ of all reference data correctly. As expected, a completely correct allocation is not achieved due to the specified outlier rate of $5 \%$. By contrast, the proportion of correctly classified test data in the defect class increases by a few percentage points by using an outlier rate. Except for case \#2 which consider only CO data $97 \%$ to $100 \%$ of the simulation data can be correctly assigned to the defect class. Within the experimental study, all data of the defect class are correctly recorded regardless of the investigation case. The different classification results between the CFD and FTIR data are mainly due to different distribution spreads.

If a particularly high value is placed on the reliable detection of defects and possible misclassifications of reference data can be accepted, the investigation case \#1 offers the best classification results, which only considerate the $\mathrm{CO}_{2}$ concentration at three different positions. However, it should be pointed out that in later applications an entire data set determines the state of an engine and not a single data point. The probabilities of success in detecting an unknown defect in the model combustion chamber are therefore very high. 


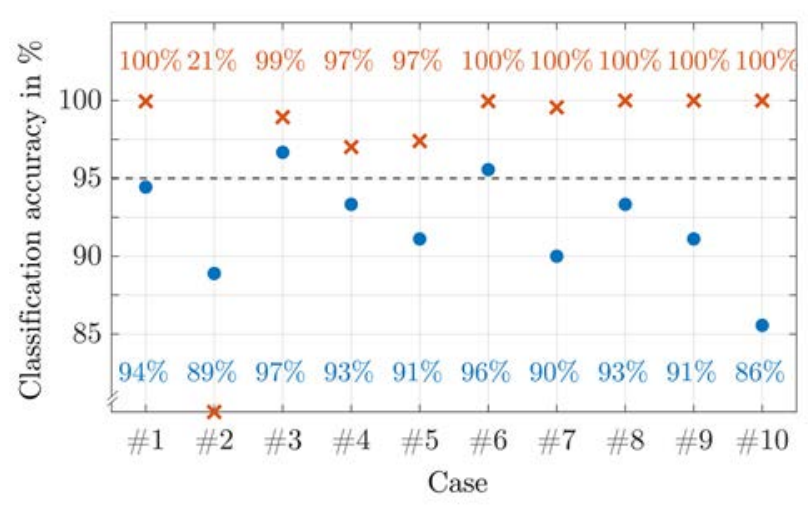

- Ref-P15ג1.0 × Defects --- Outlier rate

a) Data set from CFD simulation

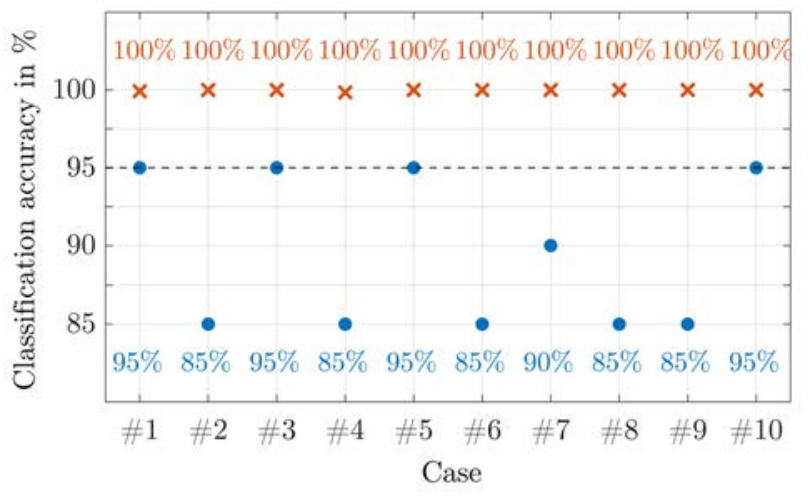

- Ref-P15ג1.0 × Defects -.- Outlier rate

b) Data set from FTIR measurement

Fig. 8: Influence of the feature selection of a one-class SVM on the proportion of correctly classified test data

\section{RESULTS: MULTI-CLASS-SVM}

In this section the results of the defect detection with a multiclass SVM are presented. In contrast to the previous section, each defect is trained as a separate class. The proportions of the correctly classified data are used to evaluate the features and model parameters of the pattern recognition. The diagrams in Fig. 9 show the percentage of correctly classified data for the features and examination cases listed in the Table 2 .

The study was carried out separately for the data sets of the CFD simulation (a) and the FTIR measurements (b). With the exception of case \#2, the classification rates are relatively high with over $85 \%$ and the trained SVM algorithm is correspondingly good. As expected, the results of the training data are higher than those of the test data. The investigations on the measurement data show higher success rates than those based on the CFD data. The reason is the lower spread of the FTIR data compared to the data sets from the simulations. It is noticeable that the classification rates for simulated data of one single position (\#4, \#5,\#6) are comparatively low. With the use of six or more features (\#7-10), the classification rate increases in both examinations to $100 \%$. The extraction position of the data $(\# 7, \# 8)$ is not relevant here. For the case $\# 9$ with a total of nine features, all data are correctly assigned.

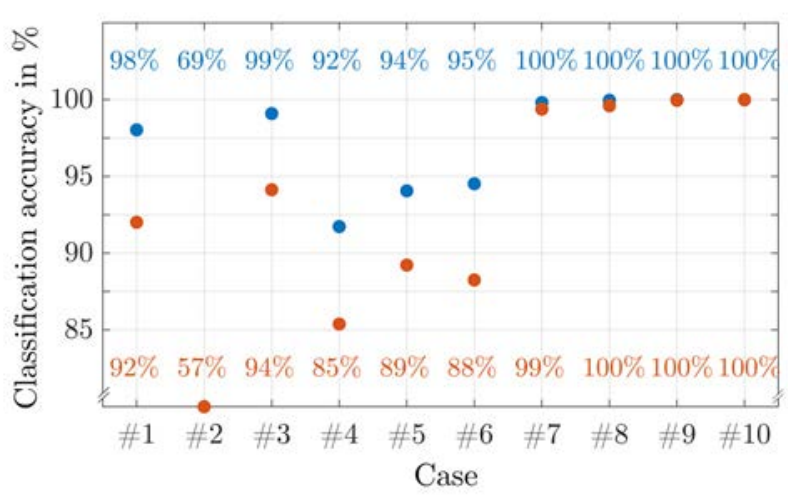

- Training data - Test data

a) Data set from CFD simulation

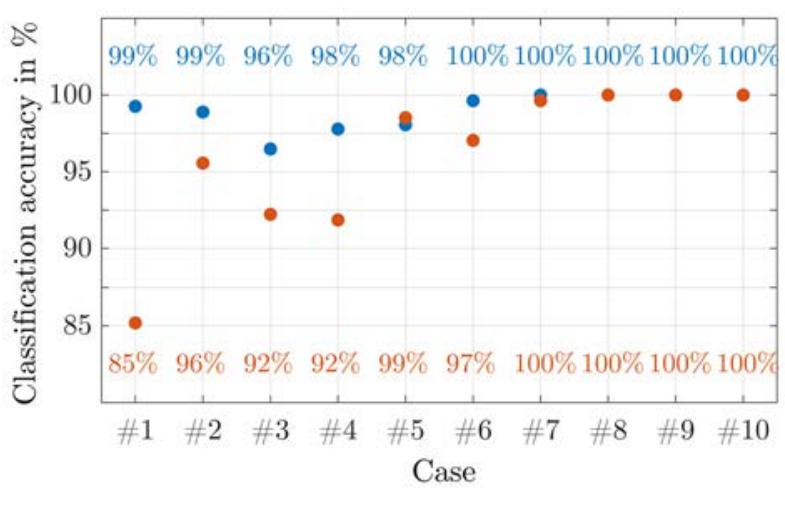

- Training data - Test data

b) Data set from FTIR measurement

Fig. 9: Influence of the feature selection of a multi-class SVM on the proportion of correctly classified data

\section{RESULTS: EXPERIMENTAL AND NUMERICAL BASED DEFECT ALLOCATION}

The next step is to replace the training data of the FTIR measurement with data sets from numerical simulations with CFD. This may reduce cost and time to implement such a system and allows to train defects, which cannot be measured before.

First studies indicate that a full classification of the measured data (testing) on basis of numerical data (training) with 23 different classes is not possible at the moment. The main reasons are the deviations between the FTIR and CFD results and the different distribution spreads. Figure 10 shows a comparison of measured

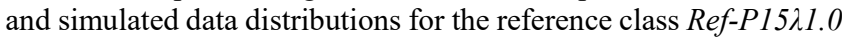
and the defect class $P 15 \lambda 0.9$. The data sets differ significantly in respect to the distribution spreads in these extreme examples. Here, the standard deviation of the simulated data is for the reference case lower, and for the defect case higher than the standard deviation of the measured data. Although the averaged results are nearly the same, different distribution spreads determine hyperplanes, which are not suitable for the training and test data and lower the classification rate.

However, the error matrix in Fig. 11 demonstrates the basic feasibility of the approach. The rows show the true class and the columns show the predicted class by the SVM algorithm. The diagonal cells (blue) correspond to data points that are correctly classified. The off-diagonal cells correspond to incorrectly (red) classified test data. The percentage of the number of test data points for every class are given in each cell. The two columns on the right sum up the proportion of correctly and incorrectly classified data for each class and row. 


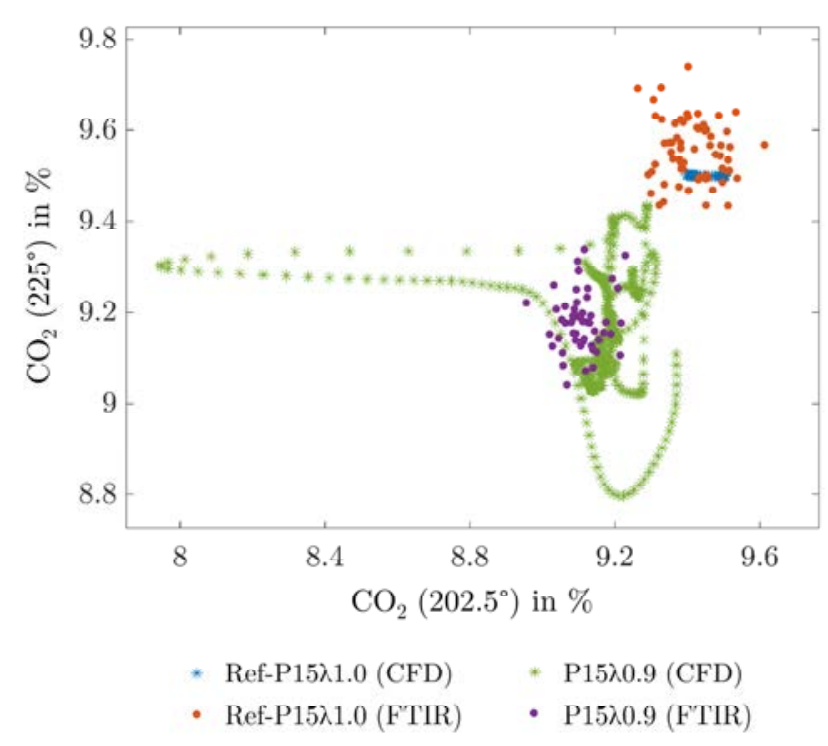

Fig. 10: Comparison of the measured and simulated data distributions for exemplary features and classes with different scatter ranges

For this classification, the number of classes is reduced to five. The investigation case $\# 1$ was used, because the measured $\mathrm{CO}_{2}$ concentration agree well with the results of the numerical

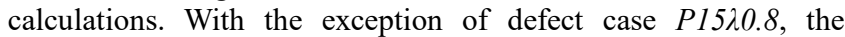
predicted classes are correct. It should be noted that different combinations of features allow to classify different defect classes correctly. For example the investigation case \#2 is useful to detect air-fuel-variations, but not for power variations.

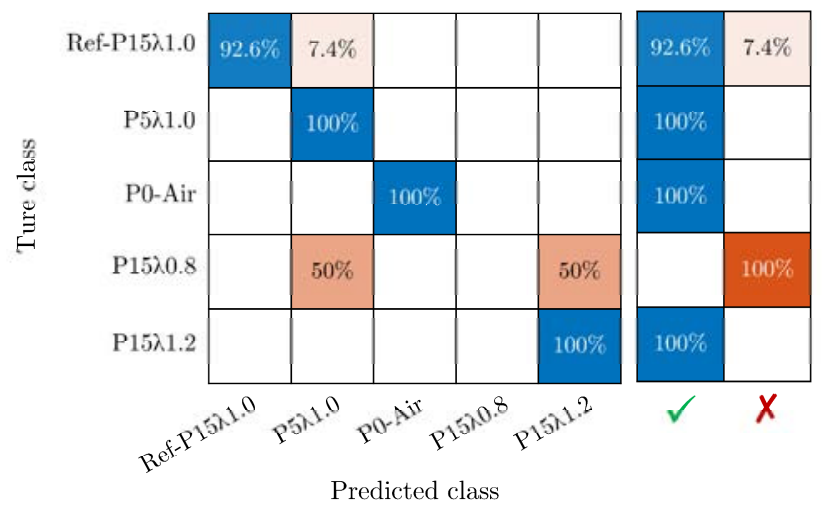

Fig. 11: Error matrix for selected classes

\section{CONCLUSION}

In order to evaluate the possibility to detect the influence of a defect burner in the exhaust plane, generic studies on a model combustor are conducted, where one out of eight swirl burners can be manipulated in its operation condition. In the current work, variations of power, air-fuel equivalence ratio and displacement of a burner are investigated, simulating a failure of this burner. With transient CFD simulations and FTIR measurements the species distribution in the exhaust plane $200 \mathrm{~mm}$ above the burner is determined. The variations are leading to a distinguishable modified pattern at the exhaust plane of the combustion chamber concerning the species distribution. The results clarify that every type of defect has a specific influence on the exhaust jet, which can be used for automatic defect detection by machine learning.

A support vector machine algorithm is implemented and trained on the simulated and measured concentration distribution of $\mathrm{CO}_{2}$, $\mathrm{CO}$ and NO. For this purpose, different combination of features are used. The study makes clear that a defect detection and defect allocation by analyzing the exhaust gases of the burner is feasible.
One-class algorithms allow to detect unknown and untrained defects, whereby multi-class algorithms can distinguish between trained defects. In both cases, all defects are detected and classified correctly. The approach of a classification on the basis of numerically generated training data could only be implemented by a reduced number of defect classes. Valid simulation models are a precondition to create training data with CFD.

In future work, it is intended to test and apply the developed SVM algorithm to numerical results of a combustor of a commercial two-shaft turbofan aircraft engine. Current investigations show, that defects affect the species and temperature distribution at the engine outlet and a defect detection seems to be feasible. Furthermore, the implementation of an experimental exhaust jet analysis on a real aircraft engine with defined combustion chamber defects is planned within the CRC 871 .

\section{ACKNOWLEDGMENTS}

The subproject A6 "Impact of Mixing on the Signature of Combustor Defects" is part of the Collaborative Research Center CRC 871 "Regeneration of complex capital goods". Funded by the Deutsche Forschungsgemeinschaft (DFG, German Research Foundation) - SFB 871/3 - 119193472

\section{REFERENCES}

[1] Paul, H., Utete, S., King, D., King, S., Anuzis, P., Tarassenko, L., 2007, „Static and dynamic novelty detection methods for jet engine health monitoring", Phil. Trans R. Soc. A, 365, 493514, doi: 10.1098/rsta.2006.1931

[2] Adamczuk, R. R., Buske, C., Roehle, I., Hennecke, C., Dinkelacker, F. and Seume J. R., 2013, "Impact of Defects and Damage in Aircraft Engines on the Exhaust Jet", Aircraft Engine; Coal, Biomass and Alternative Fuels; Cycle Innovations, Vol. 2, ASME Turbo Expo 2013: Turbine Technical Conference and Exposition, San Antonio, Texas, USA, Jun. 2013, p. V002T01A021, doi: 10.1115/GT2013-95079.

[3] Hennecke, C., von der Haar, H. and Dinkelacker, F., 2019, "Numerical Simulation of Gas Turbine Burning Chamber Combustion With Defined Burner Defects", Global Power \& Propulsion Forum Zurich19, Zurich.

[4] Hennecke, C., von der Haar, H. and Dinkelacker, F., 2017, "Failure Detection in an Annular Combustion Chamber with Experimental and Numerical Methods", Journal of Aeronautics \& Aerospace Engineering, vol. 06, no. 02, doi: 10.4172/2168-9792.1000193.

[5] Hennecke, C., 2018, "Methodik einer Zustandsbeurteilung von Triebwerksbrennkammern". Ph.D. Thesis, Leibniz University Hannover. Garbsen: TEWISS Verlag.

[6] Hartmann, U., von der Haar, H., Dinkelacker, F. and Seume, J. R., 2018, "Experimental Defect Detection in a SwirlBurner Array Through Exhaust Jet Analysis", 2018 AIAA Aerospace Sciences Meeting, Kissimmee, Florida, USA doi: $10.2514 / 6.2018-0303$.

[7] ANSYS Inc., 2017, ANSYS Fluent Theory Guide.

[8] von der Haar, H. and Dinkelacker, F., 2019, "Automatic Defect Detection in a Model Combustion Chamber Using Support Vector Machine", 9th European Combustion Meeting, Lissabon, Portugal.

[9] Boser, B. E., Guyon, I. M. and Vapnik, V. N., 1992, "A training algorithm for optimal margin classifiers" in Proceedings of the fifth annual workshop on Computational learning theory - COLT '92, Pittsburgh, Pennsylvania, United States, 1992, pp. 144-152, doi: 10.1145/130385.130401.

[10] Ladický, L. and Torr, P. H. S., 2011, "Locally Linear Support Vector Machines" in Proceedings of the Twenty-Eighth International Conference on Machine Learning, Bellevue, Washington, USA.

[11] Flechter, T, "Support Vector Machines Explained," UCL Department of Computer Science, Dec-2008. 
[12] Ben-Hur, A. and Weston, J., "A User's Guide to Support Vector Machines," in Data Mining Techniques for the Life Sciences, vol. 609, O. Carugo and F. Eisenhaber, Eds. Totowa, NJ: Humana Press, 2010, pp. 223-239.

[13] The MathWorks Inc., 2018, MATLAB (R2018b), Homepage, www.mathworks.com

\section{(C)}

Copyright (C2021 the Authors. This is an open access article distributed under the terms of the Creative Commons Attribution License, which allows reusers to distribute, remix, adapt, and build upon the material in any medium or format, so long as attribution is given to the creator. The license allows for commercial use. 